\title{
Preliminary results on a Virtual Observatory search for companions to Luyten stars
}

\author{
J. A. Caballero, F. X. Miret, J. Genebriera, T. Tobal, J. Cairol, D. Montes
}

\begin{abstract}
The Aladin sky atlas of the Virtual Observatory has shown to be a powerful and easy-handling tool for the discovery, confirmation, and characterisation of high proper-motion, multiple stellar systems of large separation in the solar vicinity. Some of these systems have very low mass components (at the star/brown dwarf boundary) and are amongst the least bound systems found to date. With projected physical separations of up to tens of thousands astronomical units, these systems represent a challenge for theoretical scenarios of formation of very low-mass stars and brown dwarfs. Here we show preliminary results of a novel "virtual" search of binary systems and companions to Luyten stars with proper motions between 0.5 and $1.0 \operatorname{arcsec} \mathrm{a}^{-1}$.
\end{abstract}

\section{Introduction}

On the one hand, Caballero (2007b) showed that a minimum of $5.0 \pm 1.8 \%$ of the solar-neighborhood dwarfs with spectral types later than M5.0 (i.e. with very low masses, $M \leq 0.1 M_{\odot}$ ) are part of multiple systems of wide separation $(r>100 \mathrm{AU})$. On the other hand, most of the objects in the New Luyten Two Tenths (NLTT) catalogue (Luyten 1979) are nearby late-type dwarfs $(d \leq 30 \mathrm{pc})$ and solar-like stars with very high proper motions. As a matter of fact, the primary stars of the two multiple systems whose common proper motions were first measured by Caballero (2007b) are two stars in the NLTT catalogue, Koenigstuhl 2A (NLTT 13422, early M) and

\footnotetext{
J. A. Caballero and D. Montes

F. X. Miret, T. Tobal and J. Cairol

Observatori Astronòmic del Garraf, Barcelona, Catalunya, Spain

J. Genebriera

Observatorio de Tacande, El Paso, La Palma, Islas Canarias, Spain
}

Departamento de Astrofísica y Ciencias de la Atmósfera, Facultad de Física, Universidad Complutense de Madrid, E-28040 Madrid, Spain, e-mail:caballeroeastrax.fis.ucm.es 
Koenigstuhl 3A (NLTT 57119, F8V). Koenigstuhl 2AB is one of the lowest mass, widest binaries yet found; Koenigstuhl $3 \mathrm{ABC}$ is the widest (hierarchical, triple) system with an L-type component $(\rho=7.530 \pm 0.007 \operatorname{arcmin}, r=11900 \pm 300 \mathrm{AU})$. Koenigstuhl 3C (also known as 2MASS J23310161-0406193 B), has a mass at the stellar-substellar boundary.

The primary star in the binary system Koenigstuhl $1 \mathrm{AB}$, discovered by $\mathrm{Ca}-$ ballero (2007a) using an identical search method, although it is too faint for appearing in the NLTT catalogue, had been also previously tabulated in a catalogue of high proper motion objects (Liverpool-Edinburgh survey; Pokorny et al. 2003). Koenigstuhl $1 \mathrm{AB}$ is the second widest system with $M_{\mathrm{A}}+M_{\mathrm{B}}<0.2 M_{\odot}(\rho=$ $1.2956 \pm 0.0012 \mathrm{arcmin}, r=1800 \pm 170 \mathrm{AU})$; the widest system, 2M0126-50 AB, was afterwards discovered by Artigau et al. (2007). The importance of systems with very low binding energies for theoretical scenarios of formation of substellar objects have been already discussed in detail in, e.g., Phan-Bao et al. (2005), Billères et al. (2005), Burgasser et al. (2007), and Artigau et al. (2007). They can also be used as a "test particle" to investigate the gravitational potential of the Galaxy (Caballero 2007a).

Given the relatively small amount of field late-type stars and brown dwarfs studied by Caballero (2007b; he investigated only 173 targets), it is natural to design a new massive survey to explore a larger quantity of dwarfs. Since the larger the proper motion of a system is, the easier the confirmation of common proper motion in a defined time base line is, one should look for companion candidates to the fastest stars. The vast majority of them were tabulated by, as the reader may guess, in the NLTT catalogue, which tabulates several tens of thousand stars with proper motions larger than two tenths arcseconds per annum $\left(\mu>0.2 \operatorname{arcsec~}^{-1}\right)$. Salim \& Gould (2003) improved the astrometry of about 36000 of these stars from (optical) USNO-A 1 and (near-infrared) $2 \mathrm{MASS} 2 \mathrm{2}$ data. Our results will complement other searches for Luyten binaries with high proper motions (e.g. Oswalt et al. 1988; Ryan 1992; Allen et al. 2000; Lépine et al. 2002; Salim \& Gould 2003; Chanamé \& Gould 2004; Levine 2005).

\section{Analysis}

We have used the survey method described in Caballero (2007a, 2007b), but with a slight difference: instead of using the SuperCOSMOS Science Archive (Hambly et al. 2001), we have employed the USNO-B13 and 2MASS catalogues and, in an intensive and extensive way, the Virtual Observatory tool Aladin (Bonnarel et al. 2000). Although the astrometric precision and depth of USNO-B1 are worst than those of SuperCOSMOS, we have still some fundamental adavantages: USNO-B1

\footnotetext{
${ }^{1}$ USNO-A: U.S. Naval Observatory catalogue of astrometric standards (Monet et al. 1998).

2 MASS: Two Micron All Sky Survey (Skrutskie et al. 2006).

${ }^{3}$ USNO-B1: U.S. Naval Observatory catalogue of astrometric standards (Monet et al. 2003).
} 


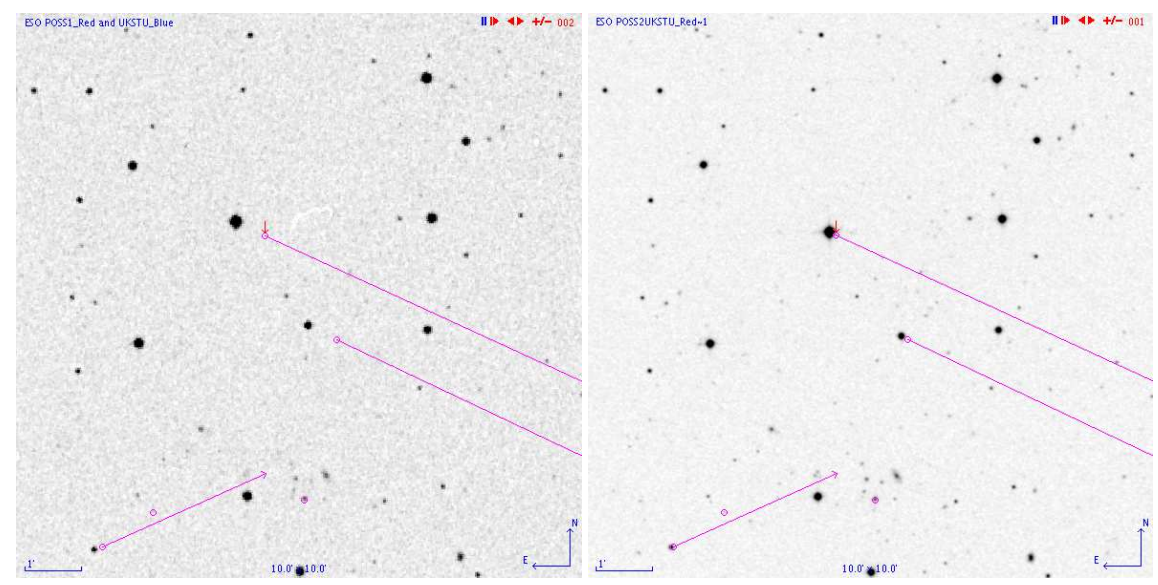

Fig. 1 Colour-inverted, $10 \times 10$ arcmin $^{2}$-size, ESO POSS1 Red (J1950.379, left) and POSS2 UKST Red (J1989.176, right) images centred on the known common proper motion system G 200-15 (NLTT 36000, sd:K4) and vB 7 (NLTT 35991, M7V). Angular separation and position angle of the system are $\rho \approx 2.21$ arcmin and $\theta \approx 214 \mathrm{deg}$. It was identified in the proper motion diagram centred on the primary. North is up and East is left. The high proper motion star to the Southeast of the system is LP 174-32, which has a different spatial velocity.

covers the whole sky (not only the Southern Hemisphere, as SuperCOSMOS does), a mathematical environment for plotting and selecting (e.g. Matlab, IDL) is unnecessary, and, especially, Aladin allows to carry out an interactive search very quickly (literally, in "a few mouse clicks"). An experienced individual with a home internet bandwidth can identify a common proper-motion companion candidate in less than two minutes. The basic scheme of the analysis is as follow:

1. choose the Luyten star to which companion candidates will be searched for. We have selected for investigation the 1947 stars (and white dwarfs) in Salim \& Gould (2003) with proper motions in the interval $0.5-1.0 \operatorname{arcsec}^{-1}$,

2. load a red $30 \times 30 \mathrm{arcmin}^{2}$ Digitized Sky Survey image centred on the star in the view window of Aladin,

3. load all the targets in the Simbad astronomical database and in the 2MASS and USNO-B1 surveys in a 15 arcmin-radius circle centred on the star,

4. cross-match 2MASS and USNO-B1 (in this order) with the default cross-matching radius of 4 arcsec,

5. identify common proper motion pairs with 2MASS+USNO-B1 data:

a. plot a proper motion diagram ( $\mu_{\delta}$ vs. $\left.\mu_{\alpha} \cos \delta\right)$ of the cross-matched sources with VOplot,

b. mark the position of the Luyten star in the diagram,

c. study if there are objects in the area with proper motions similar to those of our star,

d. if there are, check that they are not faint optical/near-infrared sources with incorrect USNO-B1 proper motions, 


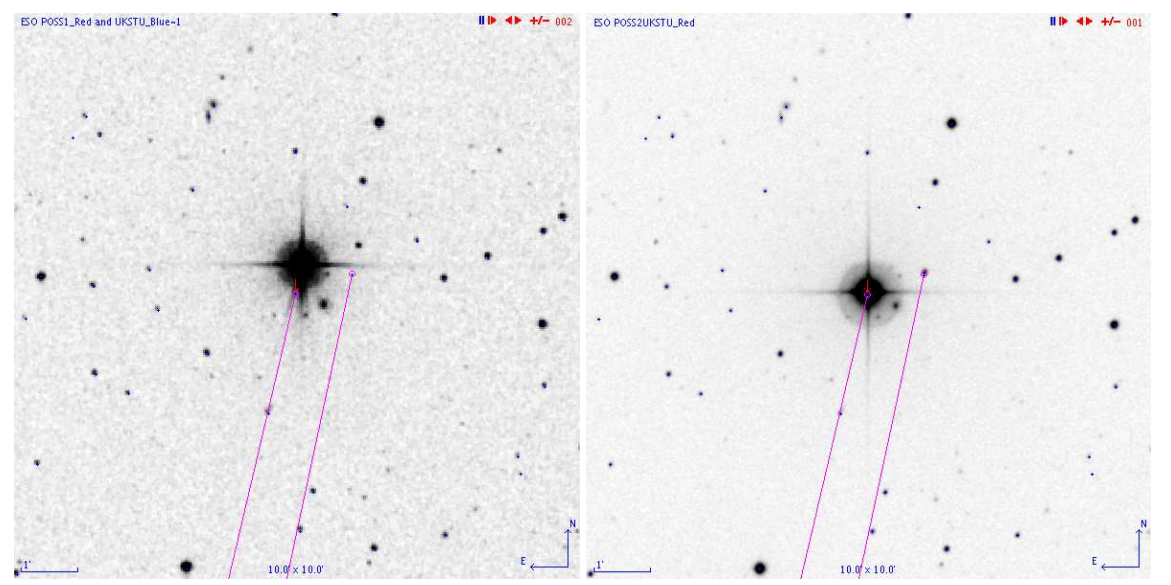

Fig. 2 Colour-inverted, $10 \times 10$ arcmin $^{2}$-size, ESO POSS1 Red (J1950.218, left) and POSS2 UKST Red (J1996.389, right) images centred on the new common proper motion system HD 126512 (NLTT 37293, F9V, $d_{\mathrm{HIP}}=46.9 \pm 1.8 \mathrm{pc}$ ) and LSPM J1425+2035W (a poorly known high proper motion star catalogued only by Lépine \& Shara 2005). Angular separation and position angle of the system are $\rho \approx 1.05$ arcmin and $\theta \approx 290 \mathrm{deg}$. It was identified with the Simbad "proper motion" filter. The secondary has the optical/near-infrared magnitudes and colours of a mid/late-K or early$\mathrm{M}$ dwarf at $d \sim 50 \mathrm{pc}$. North is up and East is left.

6. identify common proper motion pairs with Simbad data:

a. activate the "proper motion" dedicated filter,

b. study if there are objects in the area with proper motions similar to those of our star,

7. and, finally, if a common proper motion pair candidate exists, characterise it in detail. Given their brighness and wide angular separations, most of the pair candidates can be easily followed-up with small telescopes (e.g. $40 \mathrm{~cm}$ ) at amateur observatories.

We will give details on the steps of the analysis (including selection of the input list of Luyten stars, data loading and cross-matching, proper motion diagram and candidate selection, and astrometric follow-up) in a forthcoming paper.

\section{Preliminary results}

We give some preliminary results on our Virtual Observatory search for companions to Luyten stars. Of the 1947 stars in the input catalogue with proper motions in the interval $0.5-1.0 \operatorname{arcsec}^{-1}$, we have identified 101 of such stars in 52 systems identified in common proper motion diagrams. All the systems are pairs of Luyten stars except for a hierarchical triple (GJ $421 \mathrm{ABC}$ ) and four binaries with secondaries 


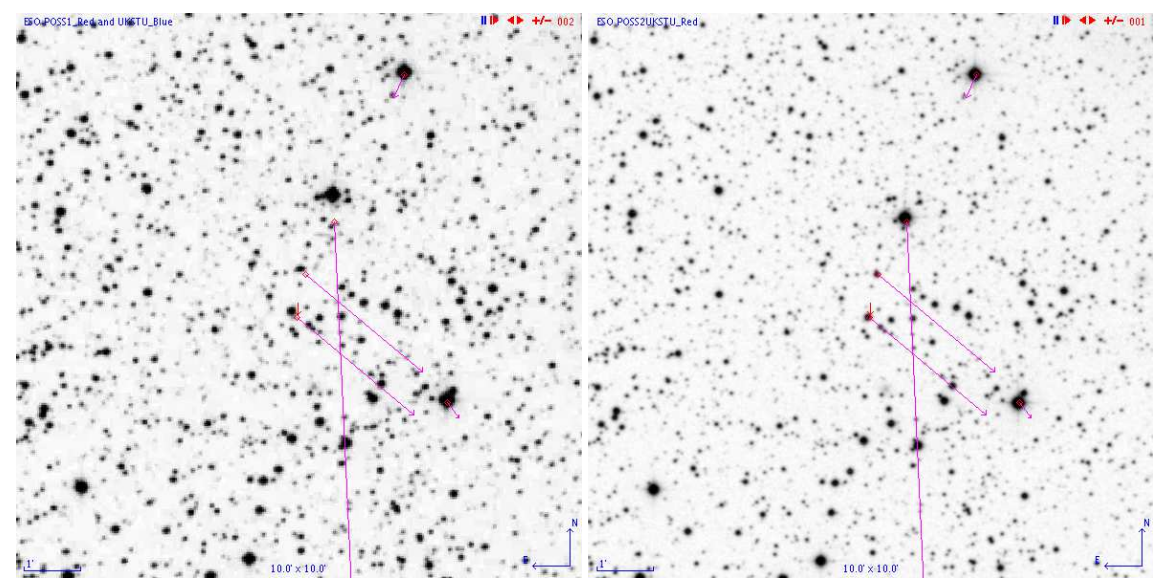

Fig. 3 Colour-inverted, $10 \times 10 \mathrm{arcmin}^{2}$-size, ESO POSS1 Red (J1952.542, left) and POSS2 UKST Red (J1992.664, right) images centred on the common proper motion system G 125-15 (NLTT 47904, M4.5, pulsation variable, X-ray) and G 125-14 (NLTT 47903, M5-6:). Angular separation and position angle of the system are $\rho=0.766 \pm 0.005$ arcmin and $\theta=347.34 \pm 0.16$ deg. The absolute value of its common proper motion, $\mu=0.16 \operatorname{arcsec} \mathrm{a}^{-1}$, prevented the system to be listed in the input catalogue. The binary was serendipitously identified in the proper motion diagram centred on the high proper motion star BD+35 3659 (NLTT 47900, F1, at about 1 arcmin to the North of the pair). North is up and East is left.

not present in Salim \& Gould (2003). From the unfinished analysis of systems preselected with the Simbad "proper motion" filter, we have identified 46 Luyten stars in 24 binaries. The primaries with no Luyten star as a secondary are GJ 383 and HD 126512 (see below). The analysis of another pre-selected $\sim 30$ Luyten stars is on-going.

The identified systems are subject to an astrometric characterisation after classyfing them into Luyten multiple systems with both components in the Hipparcos catalogue, with only one component in Hipparcos, and with no component in Hipparcos. Most of the systems in the third group have been poorly characterised or not characterised at all. Besides, we have serendipitously identified 10 additional binaries with proper motions less than $0.5 \operatorname{arcsec} \mathrm{a}^{-1}$ in the survey areas of the Luyten stars in the input catalogue.

We show in Figs. 1, 2 and 3 three examples of binary systems identified in proper motion diagrams and with the Simbad "proper motion" filter, including an interesting "serendipitous" pair. The system G $125-15+$ G $125-14$ (Fig. 31) is a bright, young, very wide, low-mass binary in the solar neighbourhood (Caballero et al., in prep.). At only $d \sim 17 \mathrm{pc}$, the most probable projected physical separation and individual masses are $\Delta \sim 800 \mathrm{AU}, M_{A} \sim 0.2 M_{\odot}$ and $M_{B} \sim 0.1 M_{\odot}$, which correspond to one of the lowest binding energies measured in a binary. It is being subject of a detailed multi-epoch, multi-band, astrometric, photometric and spectroscopic follow-up because Daemgen et al. (2007) proposed the primary to be 300-500 Ma old (i.e. individual masses could be lower). 
Acknowledgements JAC thanks Bertrand Goldman for first suggesting the extension of the Koenigstuhl survey to Luyten stars. Partial financial support was provided by the Universidad Complutense de Madrid, the Spanish Virtual Observatory, the Spanish Ministerio Educación y Ciencia, and the European Social Fund under grants AyA2005-02750, AyA2005-04286, AyA2005-24102E, and AyA2007-67458 of the Programa Nacional de Astronomía y Astrofísica and by the Comunidad Autónoma de Madrid under PRICIT project S-0505/ESP-0237 (AstroCAM).

\section{References}

1. C. Allen, A. Poveda, M. A. Herrera, $A \& A$ 356, 529 (2000).

2. É Artigau, D. Lafrenière, R. Doyon, L. Albert, D. Nadeau, J. Robert, ApJ 659, L49 (2007).

3. M. Billères, X. Delfosse, J.-L. Beuzit et al., A\&A 440, L55 (2005).

4. F. Bonnarel,, P. Fernique, O. Bienaymé et al., A\&AS 143, 33 (2000).

5. A. J. Burgasser, $A p J \mathbf{6 5 9}, 655$ (2007).

6. J. A. Caballero, $A \& A$ 462, L61 (2007a).

7. J. A. Caballero, $A p J$ 667, 520 (2007b).

8. J. Chanamé, A. Gould, ApJ 601, 289 (2004).

9. S. Daemgen, N. Siegler, I. N. Reid, L. M. Close, ApJ 654, 558 (2007).

10. N. C. Hambly, H. T. MacGillivray, M. A. Read et al., MNRAS 326, 1279 (2001).

11. S. Lépine, M. M. Shara, AJ 129, 1483 (2005).

12. S. Lépine, M. M. Shara, R. M. Rich, $A J$ 123, 3434 (2002).

13. S. E. Levine, $A J \mathbf{1 2 6}, 472$ (2005).

14. W. J. Luyten, New Luyten Catalogue of stars with proper motions larger than two tenths of an arcsecond, Minneapolis: University of Minnesota (1979).

15. D. Monet, B. Canzian, H. Harris, N. Reid, A. Rhodes, S. Sell, The PMM USNO-A1.0 Catalogue, VizieR On-line Data Catalog: I/243 (1998).

16. D. Monet, S. E. Levine, B. Canzian et al., AJ 125, 984 (2003).

17. T. D. Oswalt, P. M. Hintzen, W. J Luyten, ApJS 66, 391 (1988).

18. N. Phan-Bao, E. L. Martín, C. Reylé, T. Forveille, J. Lim, A\&A 439, L19 (2005).

19. R. S. Pokorny, H. R. A. Jones, N. C. Hambly, A\&A 397, 575 (2003).

20. S. G. Ryan, AJ 104, 1144 (1992).

21. S. Salim,. A. Gould, ApJ 582, 1011 (2003).

22. M. F. Skrutskie, R. M. Cutri, R. Stiening et al., AJ 131, 1163 (2006). 